\title{
Trattamento cronico a basse dosi con ACTH sintetico in un caso di glomerulonefrite membranosa
}

\author{
Paolo Lorusso, Emanuela Mangione, Anna Bottai, Maria Francesca Egidi \\ U.O. Nefrologia, Trapianti e Dialisi, AOUP, Dipartimento Medicina Clinica e Sperimentale, Università di Pisa, Pisa
}

\begin{abstract}
Long-term ACTH low-dose in a case of membranous nephropathy
The anti-proteinuric effect of a synthetic ACTH-analog, Tetracosactide, has been reported in membranous nephropathy resistant to previous treatments with steroids and immunosuppressive agents, as well as in other glomerulonephritis. ACTH-analog induced changes on melanocortin receptors of podocytes are the main antiproteinuric mechanisms of action. Unfortunately, recurrences of nephrotic syndrome are quite common after ACTH-analog discontinuation.

We report a case of a 20 years old female patient affected by membranous nephropathy, with nephrotic syndrome and normal renal function, which resulted resistant to steroids and cyclosporine therapy. Then she was treated by Tetracosactide (1 mg i.m. per week, for 12 months): after 6 months complete remission occurred, but a relapse was observed 6 months after the discontinuation of the therapy. A second cycle with Tetracosactide was repeated with a new complete remission after 10 months of therapy. After the one-year treatment period, ACTH-analog was continued using a maintenance dose of $1 \mathrm{mg}$ i.m. once a month: up to now, after 24 months of once-a-month Tetracosactide administration, no relapse, no side effect and no detrimental effect on renal function have been observed. This case suggests that a very low-dose and long-term Tetracosactide administration may be an effective and well tolerated therapeutic chance in patients with nephrotic syndrome resistant to steroid and/or immunosuppressive agents to avoid relapses.
\end{abstract}

Keywords: ACTH, Nephrotic syndrome, Proteinuria

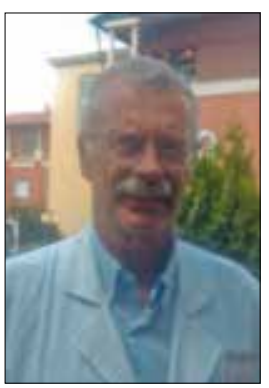

Paolo Lorusso

\section{Introduzione}

La nefropatia membranosa idiopatica è una malattia immuno-mediata con una storia naturale che va dalla remissione spontanea all'insufficienza renale terminale. Secondo alcuni Autori, il trattamento ottimale è la terapia con immunosoppressori, secondo altri il semplice controllo della sindrome nefrosica con diuretici, ACE-inibitori/ARB, statine e così via. La persistenza della sindrome nefrosica e il rapido deterioramento della funzione renale pongono generalmente l'indicazione alla terapia immunosoppressiva,

Accepted: December 3, 2015

Published online: February 5, 2016

Indirizzo per la corrispondenza:

Paolo Lorusso

U.O. Nefrologia, Trapianti e Dialisi, AOUP

Via Paradisa 2

56126 Pisa

p.lorusso@ao-pisa.toscana.it ma gli effetti collaterali di tale trattamento possono essere rischiosi, soprattutto nel soggetto anziano e "fragile".

Fino dal 1950, l'ACTH è stato efficacemente impiegato nella sindrome nefrosica nel bambino e nell'adulto (1). Tale trattamento è stato successivamente abbandonato con l'avvento della terapia con steroidi e immunosoppressori. Negli anni recenti, il trattamento con ACTH è stato riconsiderato come terapia di seconda linea nei pazienti con sindrome nefrosica, in vari quadri istologici, recidivante o resistente alla terapia con steroidi e/o immunosoppressori $(2,3)$.

L'ACTH fa parte del sistema delle melanocortine, e recettori delle melanocortine sono stati evidenziati nei podociti e nelle cellule mesangiali, endoteliali e tubulari (4).

II rene è un importante organo effettore del sistema delle melanocortine che esplicano la loro azione inibendo l'apoptosi e regolando il citoscheletro del podocita; inoltre, le melanocortine hanno un effetto antiinfiammatorio e di immunomodulazione intervenendo sui linfociti B e T.

Sono attualmente disponibili 2 formulazioni di ACTH a lento rilascio: quello sintetico (Tetracosactide) disponibile in Europa e quello naturale in gel disponibile negli USA.

Non vi sono studi di comparazione dell'effetto antiproteinurico dei due composti.

Ponticelli, in uno studio pilota randomizzato di confronto 
tra ACTH sintetico alla dose di $1 \mathrm{mg}$ i.m. 2 volte alla settimana per 1 anno e schema classico (steroide/immunosoppressore) nella nefropatia membranosa idiopatica, non ha evidenziato differenze tra i due trattamenti sull'effetto di riduzione della proteinuria (5).

L'efficacia dell'ACTH in formulazione gel nei pazienti con sindrome nefrosica resistente è stata riportata in alcuni recenti studi non randomizzati $(6,7)$. La dose cumulativa ottimale per ottenere l'effetto antiproteinurico dell'ACTH Gel è risultata di $2.800 \mathrm{UI}$, pari a $28 \mathrm{mg}(8)$.

Recentemente, il nostro gruppo ha pubblicato uno studio pilota che ha coinvolto 18 pazienti adulti con sindrome nefrosica resistente e filtrato glomerulare stimato (MDRD) superiore a $30 \mathrm{~mL} / \mathrm{min}^{*} 1.73 \mathrm{mq}$ s.c., trattati con ACTH sintetico alla dose di $1 \mathrm{mg}$ i.m. alla settimana per un anno. I quadri istologici comprendevano 10 nefropatie membranose, 3 glomerulonefriti membranoproliferative, 3 glomerulonefriti a lesioni minime e 2 glomerulosclerosi focali. Nei 16 pazienti che hanno terminato i 12 mesi di trattamento, si è verificata una remissione nel $63 \%$ dei casi, completa in 6 pazienti (38\%) e parziale in 4 (25\%). Una recidiva post trattamento si è verificata in 6 casi ( $60 \%$ delle remissioni) (9).

I risultati promettenti dell'ACTH nella sindrome nefrosica richiedono ulteriori conferme in più ampi studi randomizzati e controllati.

In questo articolo proponiamo un caso clinico che descrive un trattamento con ACTH a bassa dose, ma per un periodo molto prolungato, al fine di impedire la recidiva.

\section{Caso clinico}

Descriviamo il caso di una paziente di 20 anni affetta da sindrome nefrosica comparsa nel 2007.

All'epoca dell'esordio della sintomatologia, la paziente presentava proteinuria $7 \mathrm{~g} /$ die, disprotidemia (albuminemia $2 \mathrm{~g} / \mathrm{dL}$ e proteinemia totale $3.6 \mathrm{~g} / \mathrm{dL}$ ), funzione renale conservata (creatininemia $0.5 \mathrm{mg} / \mathrm{dL}$ ), e dislipidemia (colesterolemia totale $684 \mathrm{mg} / \mathrm{dL}$ ). L'ecografia addominale evidenziava la presenza di una falda fluida perisplenica e nello scavo pelvico con assenza di alterazioni ecostrutturali e dimensionali renali. Veniva iniziata una terapia steroidea (Deltacortene $62.5 \mathrm{mg}$ / die), proseguita per due mesi con remissione completa della proteinuria e successiva progressiva riduzione $(62.5 \mathrm{mg}$ a giorni alterni). A un mese dalla riduzione della posologia del trattamento steroideo, la paziente presentava una recidiva di sindrome nefrosica con proteinuria $7.2 \mathrm{~g} /$ die. Veniva, allora, eseguita un'agobiopsia renale che evidenziava una nefropatia membranosa idiopatica di stadio I. II trattamento per 6 mesi con ciclosporina $100 \mathrm{mg}$ x 2 die portava alla remissione completa della sindrome nefrosica. La terapia con ciclosporina veniva mantenuta fino all'Agosto del 2009. Nel Settembre dello stesso anno, a seguito di una nuova recidiva con proteinuria di $2.9 \mathrm{~g} /$ die, veniva reintrodotta la ciclosporina senza ottenere una remissione stabile della proteinuria.

Giunta alla nostra osservazione nel 2010, la paziente presentava proteinuria $6.5 \mathrm{~g} /$ die con creatininemia $0.9 \mathrm{mg} /$ $\mathrm{dL}$, eGFR MDRD $85 \mathrm{~mL} / \mathrm{min}$, albuminemia $1.8 \mathrm{~g} / \mathrm{dL}$, proteinemia totale $4 \mathrm{~g} / \mathrm{dL}$, colesterolemia totale $520 \mathrm{mg} / \mathrm{dL}$ e LDL $398 \mathrm{mg} / \mathrm{dL}$. Date le numerose recidive dopo le terapie convenzionali, veniva intrapresa una terapia con ACTH sintetico alla dose di $1 \mathrm{mg}$ i.m. alla settimana per un anno (9). Dopo 6 mesi di trattamento, si otteneva la remissione completa della sindrome nefrosica (Tab. I, Fig. 1). Tale remissione si manteneva fino al $18^{\circ}$ mese $(6$ mesi dal termine del primo ciclo), quando ricompariva una proteinuria di grado nefrosico. Si iniziava, quindi, un secondo ciclo con Tetracosactide alla stessa posologia per un anno, con remissione parziale a 6 mesi $\left(24^{\circ}\right.$ mese Tab. I, Fig. 1$)$ e completa a 10 mesi di trattamento (proteinuria $0.32 \mathrm{~g} /$ die). Da Gennaio 2013, al termine del secondo ciclo, la dose veniva progressivamente ridotta fino a un mantenimento di 1 mg i.m. al mese nell'Ottobre dello stesso anno $\left(32^{\circ} \mathrm{Tab} . \mathrm{I}\right.$, Fig. 1). La paziente prosegue tuttora questo schema terapeutico con persistenza della remissione all'ultimo followup, dopo 24 mesi di terapia di mantenimento $\left(56^{\circ}\right.$ mese Tab. I, Fig. 1). Durante il trattamento con ACTH non si sono mai osservati effetti collaterali né un peggioramento della funzione renale.

TABELLA I - Andamento di proteinuria, albuminemia, creatininemia e assetto lipidico in corso di terapia con ACTH. sono indicate le dosi di acth utilizzate: $1 \mathrm{mg} / \mathrm{s}=1 \mathrm{mg}$ alla settimana, $1 \mathrm{mg} / \mathrm{m}=1 \mathrm{mg}$ al mese

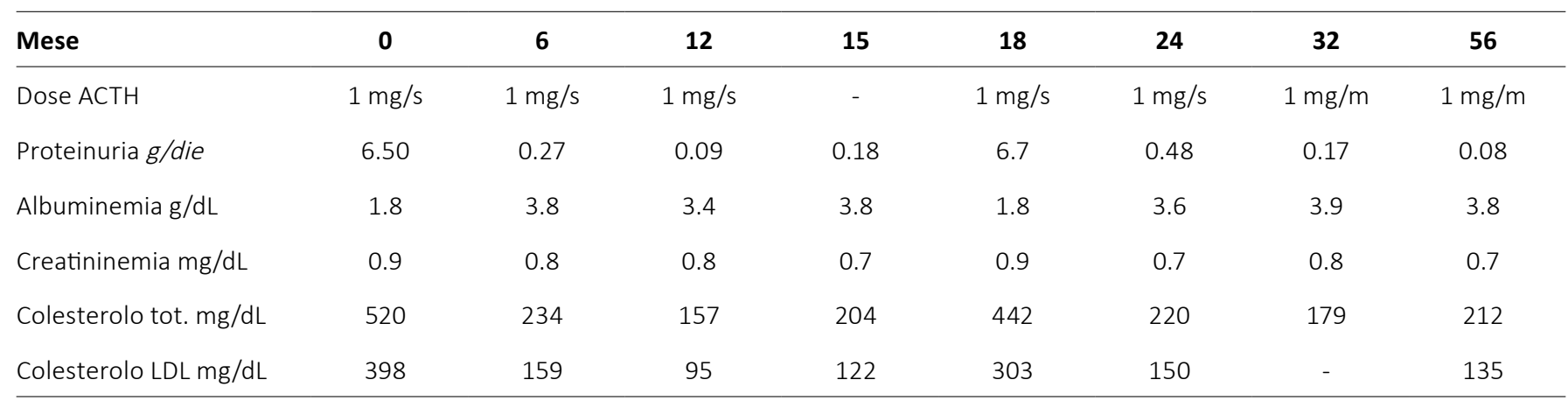




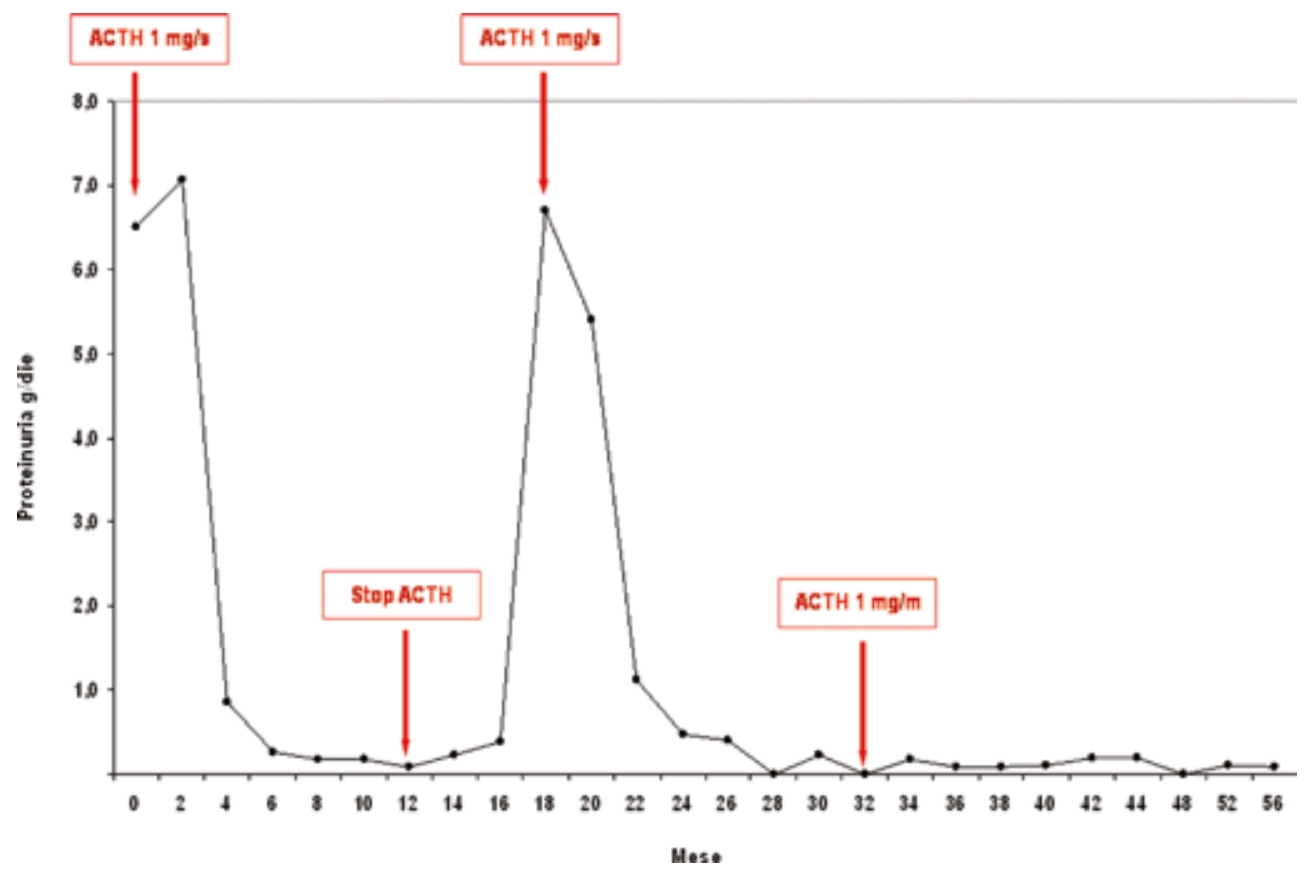

Fig. 1 - Andamento della proteinuria basale durante il follow-up. Le frecce rosse indicano l'inizio e I'interruzione della terapia con ACTH.

\section{Discussione}

La nefropatia membranosa idiopatica (NMI) può presentare un andamento cronico caratterizzato da periodi di sindrome nefrosica alternati a periodi di remissione spontanea $(30 \%)$ o farmacologica e, nel $40 \%$ dei casi, evolve verso l'insufficienza renale terminale.

Sono stati recentemente evidenziati autoanticorpi antirecettori podocitari della fosfolipasi $A 2$, responsabili della formazione dei depositi sub epiteliali che svolgono un ruolo patogenetico e predittivo nella sindrome nefrosica in corso di NMI $(10,11)$. Dati clinici e sperimentali hanno dimostrato la produzione di tali autoanticorpi da parte di cloni di linfociti B, fornendo il razionale alla terapia off label con Rituximab, che si è rivelato efficace nell'indurre remissioni cliniche nel $64 \%$ dei casi con il $30 \%$ di recidive (11), ma non esente da rischi, soprattutto per reazioni avverse alla somministrazione endovenosa.

In uno studio recente, è stata, inoltre, dimostrata una correlazione tra riduzione degli anticorpi anti-recettore della fosfolipasi A2 ed effetto antiproteinurico, indotta dall'ACTH naturale (8).

Nella nostra casistica (9), con l'uso dell'ACTH sintetico a un dosaggio settimanale di $1 \mathrm{mg}$ rispetto a $2 \mathrm{mg}$ descritto nello studio di Ponticelli (5), si è ottenuta la remissione in un $63 \%$ dei casi, di cui, però, il $60 \%$ è andato incontro a recidiva (9). L'altra casistica già citata riporta tassi di remissione più alti e di recidive più basse, ma con dosaggio più elevato e soltanto in pazienti con nefropatia membranosa idiopatica in cui I'ACTH veniva utilizzato come terapia di prima scelta (5).

Il problema delle recidive post trattamento è fondamentale, ma negli studi viene più frequentemente considerata la risposta terapeutica senza riportare l'andamento della nefropatia nei mesi e negli anni successivi o l'atteggiamento da seguire in corso di recidiva.

Per quanto riguarda la tolleranza del trattamento con ACTH sono stati riportati in letteratura, utilizzando la dose standard di 2 mg alla settimana, casi di ritenzione idrica, iperpigmentazione cutanea, intolleranza glucidica, ipertensione arteriosa e crampi muscolari. Questi effetti si sono verificati in maniera sporadica e si sono risolti alla sospensione del trattamento $(5,6,8)$. Alla posologia da noi impiegata di $1 \mathrm{mg}$ la settimana tali effetti non si sono verificati.

Dal momento che la NMI è una malattia cronica, riteniamo che sia utile trovare una posologia di mantenimento dell'ACTH da istituire dopo l'eventuale induzione della remissione.

La dose di mantenimento da noi impiegata di $1 \mathrm{mg}$ al mese, pur essendo estremamente ridotta, sembra aver protetto la paziente dalle recidive, in assenza di effetti collaterali del farmaco quali l'ipopotassiemia e la ritenzione idrica. In letteratura non vi sono studi che riportino casistiche in cui l'uso dell'ACTH sia stato protratto per oltre un anno, anche in caso di recidive.

Questo caso, anche se singolo, suggerisce la possibilità di un utilizzo della stessa dose, ma allungandone l'intervallo di somministrazione, nel tentativo di mantenere la remissione nel tempo minimizzando gli effetti collaterali.

Se confermata in una casistica più ampia, questa posologia di mantenimento potrebbe essere proposta come terapia efficace e ben tollerata della NMI per la prevenzione delle recidive, da utilizzare in sostituzione alla terapia immunosoppressiva che, impiegata per lunghi periodi, espone il paziente a gravi rischi infettivi e a neoplasie. 


\section{Disclosures}

Financial support: No financial support was received for this submission.

Conflict of interest: The authors have no conflict of interest.

\section{Bibliografia}

1. Lauson HD, Forman CW, McNamara H, Mattar G, Barnett HL. The effect of corticotropin (ACTH) on glomerular permeability to albumin in children with the nephrotic syndrome. J Clin Invest 1954;33:657-64.

2. Berg AL, Nilsson-Ehle P, Arnadottir M. Beneficial effects of ACTH on serum lipoprotein profile and glomerular function in patients with membranous nephropathy. Kidney Int 1999;56:1534-43.

3. Berg AL, Arnadottir M. ACTH-induced improvement in nephritic syndrome in patients with a variety of diagnoses. Nephrol Dial Transplant 2004;19:1305-7.

4. Gong R. Leveraging melanocortin pathways to treat glomerular diseases. Adv Chron Kidney Dis 2014;21(2):134-51.

5. Ponticelli $C$, Passerini $P$, Salvadori $M$, et al. A randomized pilot trial comparing methylprednisolone plus a cytotoxic agent versus synthetic adrenocorticotropic hormone in idiopathic membranous nephropathy. Am J Kidney Dis 2006;47:233-40.

6. Bomback AS, Tumulin JA, Baranski J, et al. Treatment of nephrotic syndrome with adrenocorticotropic hormone (ACTH) gel. Drug Des Devel Ther 2011;5:147-53.

7. Hogan J, Bomback AS, Mehta K, et al. Treatment of idiopathic FSGS with adrenocorticotropic hormone gel. Clin J Am Soc Nephrol 2013;8:2072-81.

8. Michelle A, Hladunewich MA, Cattran D, et al. A pilot study to determine the dose effectiveness of adrenocorticotrophic hormone (H.P. Acthar Gel) in nephrotic syndrome due to idiopathic membranous nephropathy. Nephrol Dial Transplant 2014;29:1570-7.

9. Lorusso P, Bottai A, Mangione E, Innocenti M, Cupisti A, Egid MF. Low-dose synthetic adrenocorticotropic hormone-analog therapy for nephrotic patients: results from a single-center pilot study. Int Nephrol Renovasc Dis 2015;8:7-12.

10. Ronco P, Debiec H. Anti-phospholipase A2 receptor antibodies and the pathogenesis of membranous nephropathy. Nephron Clin Pract 2014;128:232-7.

11. Ruggenenti $P$, Debiec $H$, Ruggiero B, et al. Anti-phospholipase A2 receptor antibody titer predicts post-rituximab outcome of membranous nephropathy. J Am Soc Nephrol 2015;26(10):2545-58.

\section{TEST DI VERIFICA}

1. In quali tipi istologici di glomerulonefrite agisce l'ACTH?
a) Solo nella glomerulonefrite membranosa
b) Solo nella malattia di Berger
c) Solo nella malattia a lesioni glomerulari minime
d) Effetto aspecifico su tutti i tipi istologici

\section{Dove si trovano i recettori renali delle melanocortine?}
a) Sui podociti
b) Sulle cellule mesangiali
c) Sulle cellule endoteliali
d) Su tutti e tre i tipi di cellule

3. Qual è la dose cumulativa di ACTH ottimale per l'effetto antiproteinurico?
a) $280 \mathrm{UI}$
b) $1120 \mathrm{UI}$
c) $2000 \mathrm{UI}$
d) $2800 \mathrm{UI}$ 